\title{
Repeatability intraexaminer and agreement in amplitude of accommodation measurements
}

\author{
B. Antona • F. Barra • A. Barrio • E. Gonzalez • \\ I. Sanchez
}

Received: 30 April 2008 /Revised: 8 August 2008 / Accepted: 13 August 2008

(C) Springer-Verlag 2008

\begin{abstract}
Background Clinical measurement of the amplitude of accommodation (AA) provides an indication of maximum accommodative ability. To determine whether there has been a significant change in the AA, it is important to have a good idea of the repeatability of the measurement method used. The aim of the present study was to compare AA measurements made using three different subjective clinical methods: the push-up, push-down, and minus lens techniques. These methods differ in terms of the apparent size of the target, the end point used, or the components of the accommodation response stimulated. Our working hypothesis was that these methods are likely to show different degrees of repeatability such that they should not be used interchangeably.

Methods The AA of the right eye was measured on two separate occasions in 61 visually normal subjects of mean age 19.7 years (range 18 to 32 ). The repeatability of the tests and agreement between them was estimated by the Bland and Altman method. We determined the mean difference (MD) and the $95 \%$ limits of agreement for the
\end{abstract}

Presentation at ARVO 2007.

This study was supported by grant PR1/07-14909 from the Universidad Complutense de Madrid.

B. Antona · F. Barra · A. Barrio · E. Gonzalez · I. Sanchez Departamento de Optica II (Optometria y Vision), Universidad Complutense,

Madrid, Spain

B. Antona $(\square)$

Escuela Universitaria de Óptica,

Universidad Complutense de Madrid,

C/ Arcos de Jalón s/n,

28037 Madrid, Spain

e-mail: bantona@opt.ucm.es repeatability study (COR) and for the agreement study (COA).

Results The COR for the push-up, push-down, and minus lens techniques were $\pm 4.76, \pm 4.00$, and $\pm 2.52 \mathrm{D}$, respectively. Higher values of AA were obtained using the pushup procedure compared to the push-down and minus lens methods. The push-down method also yielded a larger mean AA than the negative-lens method. MD between the three methods were high in clinical terms, always over $1.75 \mathrm{D}$, and the COA differed substantially by at least $\pm 4.50 \mathrm{D}$. The highest agreement interval was observed when we compared AA measurements made using minus lenses and the push-up method $( \pm 5.65 \mathrm{D})$.

Conclusions The minus lens method exhibited the best repeatability, least MD (-0.08D) and the smallest COR. Agreement between the three techniques was poor.

Keywords Accommodation amplitude - Repeatability . Agreement

\section{Introduction}

Accommodation increases the dioptric power of the eye so that the images of objects close-by can be brought into focus on the retina. The accommodation capacity of a person gradually diminishes with age and becomes clinically relevant around the age of 40 years, resulting in presbyopia. In the presbyope, the accommodation reserve is insufficient such that the subject finds it difficult to focus on near targets. Moreover, as a consequence of increasing near vision demands in modern society, accommodation problems are a common cause of vision fatigue, or ocular asthenopia, especially in adolescents and young adults [1]. In a study performed on 119 symptomatic patients of 
asthenopia aged 35 years and younger, Hokoda found that accommodative dysfunction was the most frequent condition [2]. The symptoms of asthenopia are those often related to reading or other near tasks such as: blurred vision, headache, eye tension, double vision, fatigue, difficulty in changing focus between distances and sensitivity to light [3].

In an optometric evaluation, accommodative function can be assessed by measuring the amplitude of accommodation, accommodative response, and accommodative facility. If these three factors are not assessed, then an accommodative dysfunction could pass unnoticed [4, 5]. Amplitude of accommodation (AA) measurements reflect the maximum capacity a subject has to stimulate his accommodation response. The two most widely used methods of assessing the AA are the push-up and negative lens methods. Both require that the subject correctly identify the moment at which the target fixed on becomes and stays blurred. In contrast, the push-down technique, where the patient first views a blurred stimulus, is expected to be more reliable than the push-up test in children from 5 to 9 years old [6]. Since these methods differ in terms of the end point, the apparent target size or in the accommodative components stimulated, their repeatability would be expected to differ [3].

Among the optometric methods available for determining the AA, most clinicians select one procedure for routine use depending on their personal preference. However, to determine whether a significant change has occurred in a clinical variable, it is essential to know the repeatability of the measurement procedure, and ideally we should use methods that provide measurements that are reproducible and reliable. Several studies have compared the different techniques for measuring the AA, but very few have assessed their repeatability [7-10]. The present study was therefore designed to establish the repeatability intraexaminer and agreement of measurements made with the methods most commonly used to determine AA in clinical practice: (1) the push-up method (2) the push-down method, and (3) the minus lens method.

\section{Material and methods}

\section{Study population}

The study population was comprised of 61 subjects aged 18 to 32 years (mean 19.74, SD 2.5 years) recruited from the first-year students of the School of Optics, Universidad Complutense de Madrid, Madrid, Spain. This population was selected on the grounds that: (1) the subjects were of an age with high near work demands; (2) they were unaccustomed to the type of tests performed because they were novel students, which could otherwise bias the results and not be directly extrapolated to a random clinical population of this age range. The study design fulfilled the tenets of the Declaration of Helsinki. The clinical criteria for inclusion were:

1. A corrected visual acuity (VA) greater or equal to $0.9 \quad 118$

decimal visual acuity of Snellen (20/22) in each eye at 119 distance and near. $\quad 120$

2. No eye pathology. 121

3. No history of refractive surgery, strabismus, nystagmus, 122 or amblyopia.

4. No medication or disease that could affect accommodation, fusional vergences, or ocular motility.

5. Asymptomatic subjects without accommodative or vergence alterations. The criteria used to diagnose these dysfunctions were those used in the integrative analysis approach by Scheiman and Wick [11].

Test procedures
113

114

115

116

117

0

The subjects were first subjected to a questionnaire to record their age, sex, and eye history. Next, the optometric characteristics of each subject were determined in the following tests:

- Monocular and binocular VA with and without correction. We used Snellen optotypes proyected at far vision $(6 \mathrm{~m})$ and printed at near vision $(40 \mathrm{~cm})$. Habitual correction was also recorded.

- Keratometry and objective refraction were determined using a Shin-Nippon SRW-5000 autorefractometerkeratometer. Subjective refraction was determined using the normal procedure with Snellen optotypes projected at $6 \mathrm{~m}$ and a manual phoropter [12]. Subjective refraction was performed by means of a monocular fogging method with cross-cylinder followed by binocular balancing to a standard endpoint of maximum plus for best visual acuity [12].

- Binocular vision test. Horizontal phorias were measured at distance and near using the Von Graefe technique; horizontal vergence amplitudes were determined at near and stereoacuity was evaluated using the Randot and the TNO tests. All these measurements were made with the subjective correction of the person.

132

133

134

135

136

137

138

139

140 141

According to Bland and Altman [13], the best way of 156 assessing the repeatability of an instrument is to take 157 several measurements in a series of subjects. Thus, 158 measurements were taken on two separate occasions 159 separated by a time interval of at least $24 \mathrm{~h}$. At the first 160 visit, each subject was briefly explained the objectives of 161 the study. 
There were three examiners, one for each technique. Each test was administered by the same examiner in all the subjects who was blind to the AA measurements obtained by the other methods. The tests in the two sessions were undertaken by the same examiner, so It was only studied the intra-examiner repeatability. The results of the first set of measurements were not visible during the second session, to avoid any possible influence of these on the examiner. To simulate standard clinical conditions, measurement sessions were conducted at different times of the day. Each subject underwent all the tests for one session on the same day. The order of the tests was randomly established to avoid the learning effect and/or subject fatigue affecting the results.

The AA was measured only in the right eye of each subject, while the left eye was covered. The order of the three tests: push-up, push-down, and minus lenses was randomly selected by drawing a numbered ball corresponding to each test. Each subject was requested to pick out a ball to indicate the first test and a second ball to indicate the second.

The tests were performed with the subject's distance correction. As a fixation stimulus, a near card was used with high black-on-white contrast $(\approx 80 \%)$. The card was well illuminated during the tests by keeping the column light on as well as the room lighting and it was made sure that no shadows were produced on the fixation card.

1. AA by the push-up method: This technique requires that the patient tells the examiner at which point the fixation target becomes and remains blurred. As the fixation target, we used a letter corresponding to a VA of unity, initially placed at $40 \mathrm{~cm}$. The card was then slowly moved at a speed around $5 \mathrm{~cm} / \mathrm{s}$ towards the subject's face. To maintain the subject's attention, he or she was requested to indicate when the target started to become blurred and was then requested to try to regain a clear image. The examiner stopped moving the card when the subject reported the first sustained blur. The distance was then measured (in centimeters) between the point of sustained blurring and the plane of the spectacles. By calculating the inverse of this distance (in meters) the AA was obtained in diopters.

2. AA by the push-down method: For this test, the subject viewed as the fixation stimulus, a letter corresponding to a VA of unity. The card was then gradually moved away at a speed of some $5 \mathrm{~cm} / \mathrm{s}$ from the plane of the spectacle until the letter just becomes clear and the subject could correctly identify the letter. The AA was then calculated in diopters as the inverse of the distance (in meters) from the target to the spectacle plane.
3. AA by the minus lens method: This technique consists of adding minus lenses in $0.25 \mathrm{D}$ steps while the subject views an optotype card placed at $40 \mathrm{~cm}$. The subject was asked to fix her or his sight on a letter corresponding to a VA of 0.9. A target size a little larger than for the push-up and push-down techniques was used to try to compensate, at least in part, for the reduction in size induced by minus lenses. The subject indicated the first moment when the target became and remained blurred, that is, when the subject could not focus clearly on the target by making a conscious accommodative effort. The AA could then be calculated as the sum of the added negative powers over subjective refraction plus $2.50 \mathrm{D}$, which corresponds to the accommodative effort needed to clearly view the target at a working distance of $40 \mathrm{~cm}$.

Once the data had been collected for the whole sample they were processed statistically. Data analysis was performed using the Analyse-it for Microsoft Excel (Leeds, UK. See http://www.analyse-it.com) and SPSS (v. 11 for Windows, SPSS Inc., Chicago, IL, USA).

The Bland and Altman method was used to determine the degree of repeatability and agreement of the tests [13, 14]. From a clinical perspective, the advantage of this method is that the agreement of the tests is expressed in the same units of measurement as the test itself and allows the clinician to establish his own criteria as to whether or not a difference is significant. This method was used when the differences, as established by the Anderson-Darling normality test, showed a normal distribution.

The factors determined were the mean difference, the standard deviation (SD), the coefficient of repeatability $(\mathrm{COR}=1.96 \times \mathrm{SD})$ and the limits of agreement at the $95 \%$ level (mean difference $\pm \mathrm{COR}$ ). The $t$-test for paired samples was also used to establish the significance of the differences observed. The level of significance was set at $p<0.05$. When the normality test revealed a non-normal distribution, instead of calculating the COR, we determined the 95th percentile of the absolute values of the differences. Similarly, we determined the coefficient of agreement (COA) among the tests.

Given the sample size, a small difference could be statistically significant yet not clinically significant. Differences from the mean were plotted to establish the limits of agreement at the 95\% level and obtain a better idea of the repeatability of the measures. The limits of the agreement interval constitute a threshold for the differences in successive measures that have to be surpassed if the difference indicates that a change in the value has in effect 
occurred and cannot simply be explained by natural variation among measurements.

\section{Results}

\section{Repeatability}

Figure 1 shows difference vs. mean plots (Bland-Altman) with the difference between two measures (final - initial) on the $y$-axis plotted against the average of the two measures on the $\mathrm{x}$-axis. For each plot, if measures show good repeatability, the averaged difference will be close to 0 , and the $\pm 1.96 \mathrm{SD}$, or the $95 \%$ limits of agreement will be small. No one of those plots shows a tendency for the difference to increase with the dioptric value, i.e., the repeatability of the tests does not change with accommodative amplitude. Table 1 shows the repeatability of the results obtained for the three methods of measuring the AA. The minus lens method has the highest repeatability, since it shows the least mean difference $(\mathrm{MD}=-0.08 \mathrm{D})$ and smallest $95 \%$ agreement interval $(\mathrm{COR}= \pm 2.52 \mathrm{D})$.

\section{Agreement}

Bland and Altman [13] have also suggested that rather than determining correlation coefficients, a more informative method of comparing the results of two methods of testing is to plot the differences between the results obtained by the two methods against the mean of the two methods. When this is done, the mean of the results are plotted along the $\mathrm{x}$ axis, and the differences are plotted along the y-axis. Horizontal lines are plotted, indicating the mean of the differences (MD) and the limits of agreement between the two methods, $\mathrm{MD} \pm 1.96 \times \mathrm{SD}$ of the differences. Such plots for our data of accommodative amplitude are shown in Fig. 2. The COA are sufficiently high so that the two methods could not be used interchangeably. None of those plots shows a tendency for the difference to increase with the amplitude value, i.e., the level of agreement between tests does not change with the amplitude of accommodation range

Table 2 shows the results of our analysis of agreement among the different ways of measuring the AA. Significant differences in agreement emerged among the three measurement methods. Both the mean differences and agreement levels were high, especially when comparing the minus lens method with the push-up test $(\mathrm{COA}= \pm 5.65 \mathrm{D})$.

As a complement to the Bland and Altman method, we also conducted a two-factor (method and session) repeated measures ANOVA. This method revealed significant differences among the tests $\left(\mathrm{F}_{2,120}=110.45\right.$; $p<0.0001)$ and between the two measurement sessions

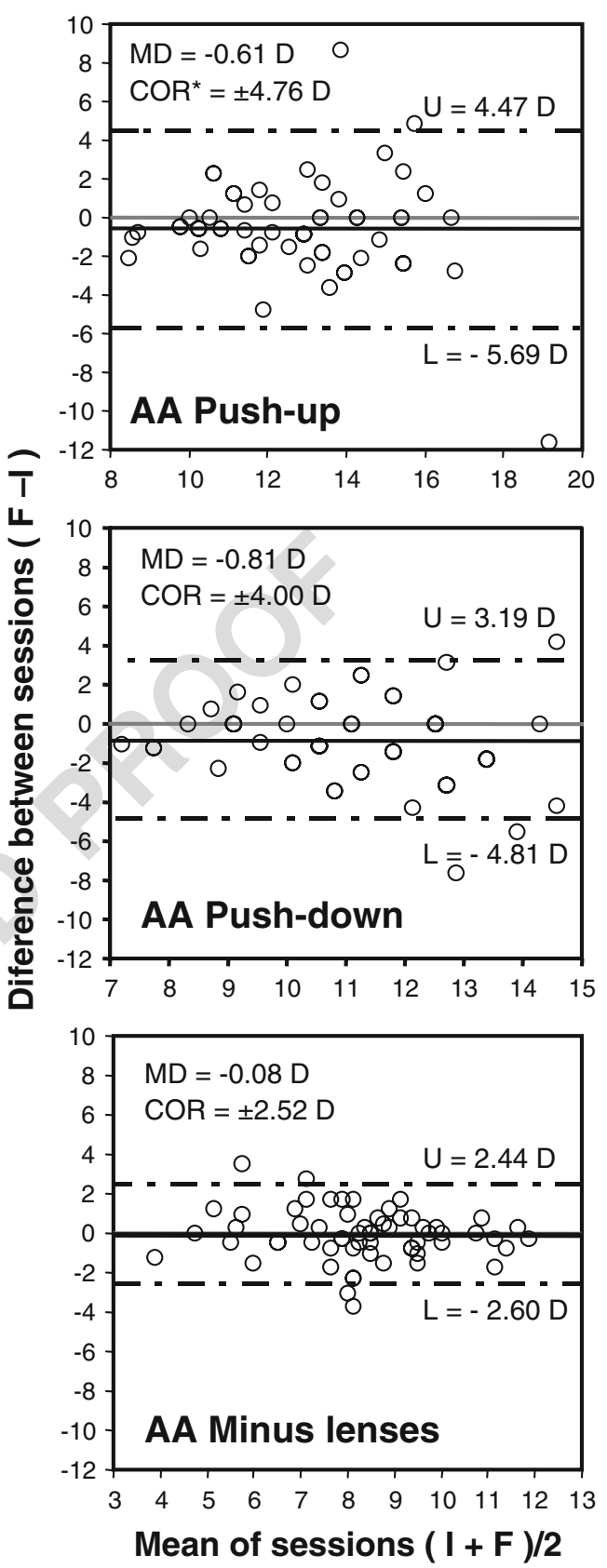

Fig. 1 Difference vs. mean plots of repeatability for accommodative amplitude measurements. The solid line represents the averaged difference on the measurements between final session and initial session (mean difference, MD). The dotted lines indicate the lower (L) and the upper (U) $95 \%$ limits of agreement $(\mathrm{MD} \pm 1.96 \times \mathrm{SD})$. When the distribution of differences was not normal, it is included the 95th percentile of the absolute differences (COR*)

$\left(\mathrm{F}_{1.60}=7.76 ; p=0.007\right)$. However, no interaction effects between the two factors were detected $\left(\mathrm{F}_{2,120}=2.74 ; p=\right.$ 0.07). A post hoc analysis using the Scheffé technique indicated significant differences between each of the mean AA values obtained using the push-up, push-down, and minus lens procedures. The mean AA recorded using the minus lens procedure $(8.56 \mathrm{D})$ was lower than using the 
t1.1 Table 1 Repeatability in amplitude of accommodation (AA) measurements

\begin{tabular}{llllllll}
$\mathrm{t} 1.2$ & & Mean (D) & SD (D) & MD (F-I) (D) & p (test-t) & COR (D) & p (A-D) \\
\cline { 2 - 7 } t1.3 & AA by push-up & 13.08 & 2.79 & -0.61 & $\mathrm{p}=0.002$ & $\pm 4.76^{*}$ \\
$\mathrm{t} 1.4$ & AA by push-down & 11.25 & 1.77 & -0.81 & $\mathrm{p}=0.06$ & \pm .0002 \\
$\mathrm{t} 1.5$ & AA by minus lenses & 8.56 & 1.72 & -0.08 & $\mathrm{p}=0.6$ & \pm 4.00 & \pm 2.52 \\
\cline { 2 - 6 }
\end{tabular}

t1.6 Key: $\mathrm{D}=$ diopters, $\mathrm{SD}=$ standard deviation, $\mathrm{MD}=$ mean difference, $\mathrm{F}=$ final, $\mathrm{I}=$ initial, $\mathrm{COR}=$ coefficient of repeatability $\mathrm{p}(\mathrm{A}-\mathrm{D})=\mathrm{p}$ (normality test of Anderson-Darling)

*COR replaced by the 95 th percentile of the absolute differences

319 push-down method (11.25 D), which was in turn lower

320 than that obtained using the push-up protocol (13.08 D).

\section{Discussion}

\section{Repeatability}

When measuring AA using subjective methods, the COR should be determined since it establishes the minimum change in the AA that clearly surpasses the margin of error inherent to the technique for each age range. The smallest margin of error, or lowest COR, was obtained here for the minus lens method in which a change of up to $\pm 2.52 \mathrm{D}$ may be attributed to measurement errors and not to a real change in the AA.

Rosenfield and Cohen [8] assessed the repeatability of the same three methods of measuring AA in a population of 13 subjects of similar age to our participants $(24.3 \pm$ 0.5 years). Their results indicated agreement intervals (CORs) significantly improved over the ones we recorded (push-up: $\pm 1.44 \mathrm{D}$, push-down: \pm 1.39 , minus lenses: \pm 1.43 D) and the three methods showed a similar degree of repeatability. Among the factors possibly contributing to this discrepancy are the smaller measurement sample and the way in which the mean standard deviation for the sample was calculated, from which the authors then estimated the agreement interval. Subjects were attended for a total of five sessions for each method and three measurements was taken in each session. For every subject, mean and standard deviation was calculated without considering different sessions. Then, the agreement interval for each method was calculated from the mean of all the individual standard deviations. We would expect this method to filter out extreme measures. In addition, their small sample size of 13 subjects will diminish the statistical power of the results. Another factor that could also increase variability of our results is the fact the measurements were taken at different times of the day.

Chen and O'Leary [9], using the push-down method in two separate sessions on a sample of 18 adults (18 to 19 years), found a mean difference and 95\% limits of agreement of $0.07 \pm 1.24 \mathrm{D}$. Rouse et al. [10] assessed the intra-examiner repeatability of the push-up method for measuring the amplitude of accommodation in 20 fifth and sixth grade school children reported an intra-examiner between-session repeatability differed by examiner $( \pm 5.32$ $\mathrm{D}$ and $\pm 10.48 \mathrm{D})$. Our results $( \pm 4.76 \mathrm{D})$ are closer to those reported by Rouse et al. [10] although it must be considered that they used a younger sample of subjects and the COR may be expected to be higher in a younger population.

We have evaluated the repeatability intra-examiner where all of the testing for each method was carried out by the same examiner. When performing repeatability studies or studies that compare different methods to measure the same ability, it is very important to implement a good masking strategy and to apply the tests in random order to asegurate the independence in the measures [15]. If different examiners carry out successive tests, as in this study, it will asegurate good masking of the examiner but this will give rise to another source of variance which is likely to inflate the error variation in difference between scores obtained on the two visits. However, with subjective tests if all the different examiners apply the tests following exactly the same protocol and giving the same instructions to the patient, probably the increase in variation associated to different examiners will not be very high in comparison to objective tests where the examiner's intervention is decisive [16]. Nevertheless, future studies where all three of the methods measuring the amplitude of accommodation performed by the same clinician are necessary to probe this aspect.

Agreement

Our results confirm those of other studies indicating that the minus lens method usually provides lower AA values than the other two methods (see Table 1). This difference may be attributed to the reduction in target size induced by minus lenses that increases the subject's ability to first detect blur giving rise to a lower AA measurement [17]. On the other hand, the absence of proximal cues in the minus-lens technique could contribute to the smaller AA achieved using this method. For example, even though the subjects' left eyes are occluded, changes in the physical distance of the target could produce proximal vergence that is absent in 

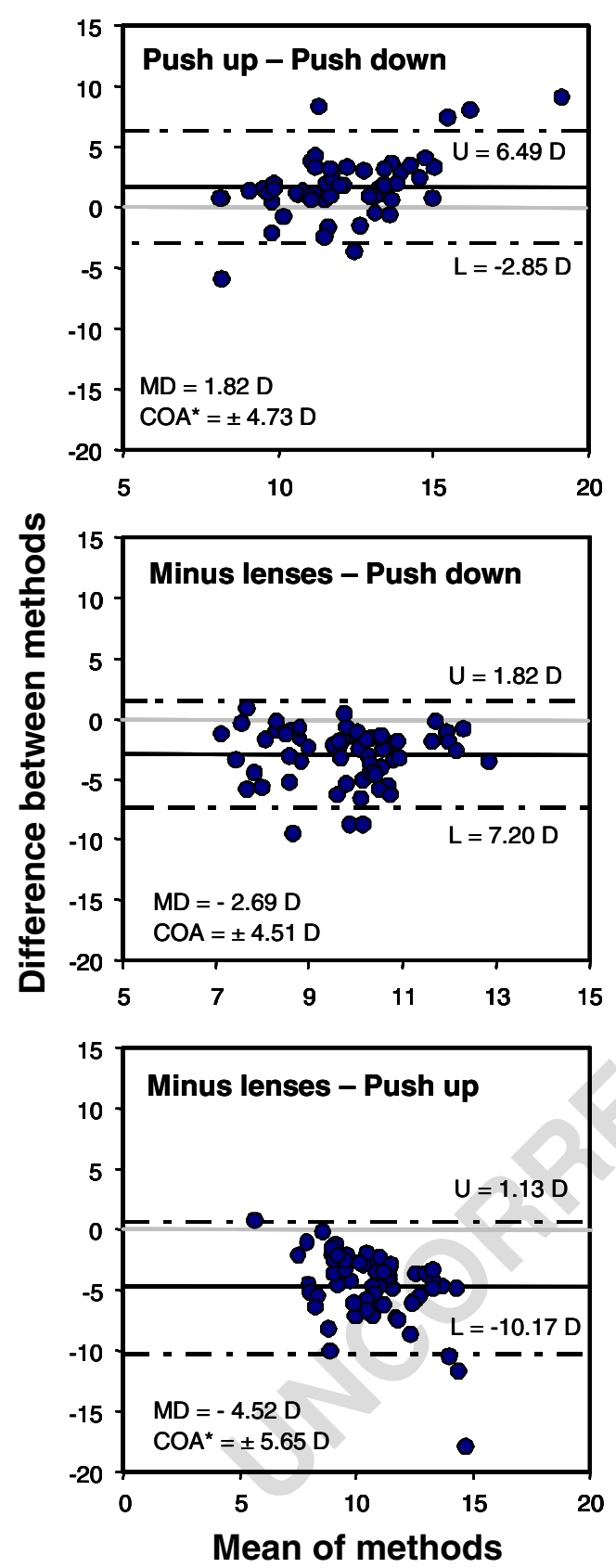

Fig. 2 Difference vs. mean plots of method comparisons. The solid line represents the averaged difference (MD) of the measurements between different methods. The dotted lines indicate the lower $(\mathrm{L})$ and the upper (U) $95 \%$ limits of agreement $(\mathrm{MD} \pm 1.96 \mathrm{SD})$. When the distribution of differences was not normal, it is included the 95th percentile of the absolute differences (COA*) the minus-lens technique. This proximal vergence could contribute to the accommodative amplitude via the $\mathrm{CA} / \mathrm{C}$ crosslink, in addition to any proximal accommodation that is stimulated directly [1]. According to our findings, the push-down method would be expected on average to show an AA 2.69 D higher than the minus lens method and the push-up method an AA 4.52 D higher than the minus lens method. Several studies have compared the mean AAs obtained using the push-up and minus lens methods, the latter procedure always yielding the lowest values although not as low as those obtained here [18]. For example, Rambo and Sangal [19] noticed a difference of 1.49 D for a study population of mean age 27.5 years, while Kragha [20] recorded a difference of $1.23 \mathrm{D}$ for a group of subjects aged 23 to 27 years.

Atchison et al. [17] claim that the push-up and pushdown methods offer a clinically more useful measurement of AA than that provided by minus lenses, since they inform the clinician of the near point of clear vision. Notwithstanding, when using a fixed-size target, the pushup method tends to overestimate the AA due to the apparently increased target size and to greater stimulation of proximal accommodation [1]. This overestimate of the AA is more marked in young subjects with a high AA than for presbyopes and pre-presbyopes, while the push-up method may be an overestimate of even other subjective AA measurements, subjective measures tend to overestimate true accommodative amplitude measured objectively by clinical autorefractors [21]. The fact that the push-up method provides higher AA values than the push-down or minus lens methods has been reported previously [22, 23]. Studies in which the push-up method using a single chart were compared to methods free from the effect of an increased depth of focus have established this overestimation of AA to be between 1.50 and $2.50 \mathrm{D}$ [1, 3, 17, 24]. This finding has no significant clinical implications for subjects with a high AA, yet in young presbyopes or prepresbyopes (35-45 years), any overestimate could lead to a near addition lower than really needed or to a lack of prescription in a subject who would benefit from plus lenses.

We would expect the push-down method to render lower AA values than the push-up procedure, possibly because the influence of two aspects: First, because the two
399

400 401 402 403 404 405 406 407 408 409 410 411 412 413 414 415 416 417 418 419 420 421 422 423 424 425 426 427 428 429 430 431 432 433 434 435 436 437 438

t2.1 Table 2 Agreement between the tests used to measure the amplitude of accommodation

\begin{tabular}{|c|c|c|c|c|}
\hline & MD (D) & $\mathrm{p}$ (test-t) & $\mathrm{COA}(\mathrm{D})$ & $\mathrm{p}(\mathrm{A}-\mathrm{D})$ \\
\hline AA push-up - AA push-down & +1.82 (up > down) & $p<0.0001$ & $\pm 4.73 *$ & 0.0001 \\
\hline AA minus lenses - AA push-down & -2.69 (down $>$ lenses $)$ & $p<0.0001$ & \pm 4.51 & 0.06 \\
\hline AA minus lenses - AA push-up & -4.52 (up > lenses) & $p<0.0001$ & $\pm 5.65^{*}$ & 0.0004 \\
\hline
\end{tabular}

t2.6 Key: $\mathrm{D}=$ diopters, $\mathrm{MD}=$ mean difference, $\mathrm{COA}=$ coefficient of agreement, $\mathrm{p}(\mathrm{A}-\mathrm{D})=\mathrm{p}$ (normality test of Anderson-Darling) $*$ COA replaced by the 95 th percentile of the absolute differences 
procedures use different endpoints: the push-up technique measures the first blur, whereas the push-down procedure determines the first clear point, closer to the eyes. Second, in the push-up procedure, the subject is aware of the test letter from the start and will find it easier to identify despite it being blurred. On the contrary, with the push-down method, the subject is unaware of the test letter and will therefore take longer to give a correct reply. These effects were reflected in our results, the mean push-up AA being $1.82 \mathrm{D}$ higher than the push-down AA and in the study of Chen and O'Leary [9], that on a sample of 27 subjects ( 9 to 39 years, mean: 19,53), found a mean difference of $1.40 \mathrm{D}$.

In conclusion, amplitude of accommodation measurements made using the minus lens method exhibited the best repeatability, lowest mean difference $(-0.08 \mathrm{D})$ and smallest $95 \%$ interval of agreement $( \pm 2.52 \mathrm{D})$. This last variable was notably improved over the agreement intervals recorded for the push-up $( \pm 4.76 \mathrm{D})$ and push-down $( \pm 4.00$ D) techniques.

Our results indicate that the push-down method provides a mean AA 2.69 D higher, and the push-up method one 4.52 D higher, than measurements made using minus lenses. Agreement between the three techniques was poor, indicating that the methods are not interchangeable.

Acknowledgements This study was supported by grant PR1/0714909 from the Universidad Complutense de Madrid.

\section{References}

1. Rosenfield M, Gilmartin B (1990) Effect of target proximity on the open-loop accommodative response. Optom Vis Sci 67:74-79

2. Hokoda SC (1985) General binocular dysfunctions in an urban optometry clinic. J Am Optom Assoc 56:560-562

3. Rosenfield M (1997) Accommodation. In: Zadnik K (ed) The ocular examination; measurements and findings. WB Saunders, Philadelphia

4. Wick B, Hall P (1987) Relation among accommodative facility, lag, and amplitude in elementary school children. Am J Optom Physiol Opt 64:593-598

5. Goss D (1992) Clinical accommodation testing. Curr Opin Ophthalmol 3:78-82. doi:10.1097/00055735-199202000-00011

6. Woehrle MB, Peters RJ, Frantz KA (1997) Accommodative amplitude determination: can we substitute the pull-away for the push-up method? J Optom Vis Dev 28:246-249
7. Brozek J, Simonson E, Bushard WJ, Peterson JH (1948) Effects of practice and the consistency of repeated measurements of accommodation and vergence. Am J Ophthalmol 31:191-198

8. Rosenfield M, Cohen AS (1996) Repeatability of clinical measurements of the amplitude of accommodation. Ophthalmic Physiol Opt 16:247-249. doi:10.1016/0275-5408(95)00093-3

9. Chen AH, O'Leary DJ (1998) Validity and repeatability of the modified push-up method for measuring the amplitude of accommodation. Clin Exp Optom 81:63-71

10. Rouse MW, Borsting E, Deland PN (2002) Reliability of binocular vision measurements used in the classification of convergence insufficiency. Optom Vis Sci 79:254-264. doi:10.1097/00006324-200204000-00012

11. Scheiman M, Wick B (2002) Clinical management of binocular vision. Heterophoric accommodative and eye movement disorders. Lippincott Williams \& Wilkins, Philadelphia

12. Carlson NB, Kurtz D (2004) Clinical procedures for ocular examination. McGraw Hill, New York

13. Bland J, Altman DG (1986) Statistical methods for assessing agreement between two methods of clinical measurement. Lancet 1-8476:307-310

14. Zadnik K, Mutti DO, Adams AJ (1992) The repeatability of measurement of the ocular components. Invest Ophthalmol Vis Sci 33:2325-2333

15. Argimon JM, Jimenez J (2004) Métodos de investigación clínica y epidemiológica. Harcourt, Madrid

16. Reeves BC, Hill AR, Aspinall PA (1987) The clinical significance of change. Ophthalmic Physiol Opt 7:441-446

17. Atchison DA, Capper EJ, McCabe KL (1994) Critical subjective measurement of amplitude of accommodation. Optom Vis Sci 71:699-706. doi:10.1097/00006324-199411000-00005

18. Ostrin LA, Glasser A (2004) Accommodation measurements in a prepresbyopic and presbyopic population. J Cataract Refract Surg 30:1435-1444. doi:10.1016/j.jcrs.2003.12.045

19. Rambo VC, Sangal SP (1960) A study of the accommodation of the people of India. With further notes on the development of presbyopia at different ages in different peoples. Am J Ophthalmol 49:993-1004

20. Kragha IKOK (1989) Measurement of amplitude of accommodation. Ophthalmic Physiol Opt 9:342-343. doi:10.1111/j.1475-1313.1989. tb00925.x

21. Wold JE, Hu A, Chen S, Glasser A (2003) Subjective and objective measurement of human accommodative amplitude. J Cataract Refract Surg 29:1878-1888. doi:10.1016/S0886-3350 (03)00667-9

22. Fitch RC (1971) Procedural effects on the manifest human amplitude of accommodation. Am J Optom Arch Am Acad Optom 48:918-926

23. Hokoda SC, Ciuffreda KJ (1982) Measurement of accommodative amplitude in amblyopia. Ophthalmic Physiol Opt 2:205-212

24. Rosenfield M, Cohen AS (1995) Push-up amplitude of accommodation and target size. Ophthalmic Physiol Opt 15:231-232 letter. doi:10.1016/0275-5408(95)90576-N 